DOI: 10.36868/ejmse.2020.05.03.145

\title{
THE ROLE OF ORGANOSILICATES ON THE DIELECTRIC RELAXATIONS OF BIOMEDICAL THERMOPLASTIC POLYURETHANE NANOCOMPOSITES
}

\author{
Azlin Fazlina OSMAN ${ }^{1,2 *}$, Darren J. MARTIN ${ }^{2}$, James RUNT ${ }^{3}$ and \\ Pornpen ATORNGITJAWAT ${ }^{3,4}$
}

\begin{abstract}
${ }^{1}$ Center of Excellence Geopolymer and Green Technology (CEGeoGTech), School of Materials Engineering, Universiti Malaysia Perlis, Arau, Perlis 02600, Malaysia.

${ }^{2}$ Austra lian Institute for Bioengineering and Nanotechnology, The University of Queensland, Building 75, Cnr College and Cooper Rds, Brisbane, 4072, Australia.

${ }^{3}$ Department of Materials Science and Engineering, The Pennsylvania State University, University Park, Pennsylvania, 16802 , USA.

${ }^{4}$ Department of Chemistry, Faculty of Science, Burapha University, Chonburi 20131, Thaila nd.
\end{abstract}

\begin{abstract}
The molecular dynamics of a series of biomedical poly(dimethylsiloxane)(PDMS)-based thermoplastic polyurethane nanocomposites (TPU) containing low and high aspect ratio organosilicates was investigated using broadband dielectric spectroscopy (DRS). The microphase separated morphology and degrees of separation, extensively characterized in a previous publication, were used to aid the interpretation of the molecular dynamics. Three relaxations were identified in dielectric spectra of the neat host TPU and nanocomposites over the measured temperature and frequency range: the low temperature process associated with local motion of the PDMS phase $\left(\alpha_{1}\right)$, the higher temperature process which is associated with the mixed phase segmental motion $\left(\alpha_{2}\right)$, and Maxwell-Wagner-Sillars (MWS) interfacial polarization $\left(\alpha_{3}\right)$ process. The incorporation of organoclays has previously been shown to influence the morphology of these TPU systems, consequently affecting their dielectric behaviour. In this work the segmental relaxation time and temperature of $\alpha_{1}, \alpha_{2}$, and $\alpha_{3}$ process of the TPU are significantly altered by the presence of the low aspect ratio organo-hectorite. These significant changes in the TPU dynamics were due to very strong interactions bet ween the exfoliated organo-hectorite and the TPU matrix, with the low aspect ratio nanofiller effectively acting as an interfacial compatibilizer. The dielectric spectroscopy data confirmed the existence of phase mixing in the E5-325 TPU containing organo-hectorite.
\end{abstract}

Keywords: thermoplastic polyurethane, nanocomposites, organosilicates, dielectric spectroscopy. relaxation

\section{Introduction}

In today's world, thermoplastic polyurethanes (TPUs) are one of the most versatile materials. TPUs have increasingly been used in a wide range of applications for several decades because they offer the widest variety of chemical and physical properties [1]. TPUs have found extensive use in numerous commercial applications such as adhesives, sport equipments, synthetic leathers, coatings, sealants, foam, membranes, foams and biomedical applications because of the wide range of compositions possible [1-3]. The poly(dimethylsiloxane) (PDMS) / poly(hexamethylene oxide) (PHMO)-based TPUs represent potential candidates for biomedical application due to their exceptional mechanical properties, biocompatibility and biostability $[4,5]$. This PDMS/PHMO based TPUs may have high potential to achieve immense physical, 
mechanical and thermal properties when added with a little amount of nanofiller, thus have a great deal of interest in recent years [5-7].

The morphology of the PDMS/PHMO-based TPU is quite complex due to the multiphase structure and chemical heterogeneity induces by immiscibility of the mixed soft segment $[4,5]$. The macroscopic and microscopic properties depend on the nature of both soft and hard phase and the degree of phase mixing between both domains [4,5]. The addition of the chemically modified nanosilicate into this TPU system increases the complexity of the structure. It is generally accepted that the improved properties of polymer nanocomposites are related to modification of the structure and molecular dynamics of the polymer due to the interaction with the filler surface [8-14]. A better understanding of these relationships may provide a basis for understanding for tailoring and improving the properties at the molecular level.

Broadband Dielectric Relaxation Spectroscopy (DRS) analysis is an established technique to study the molecular dynamics of polymers due to the fact that response over a broad frequency range from the milli to mega Hertz region can be tested. This broad frequency range allows us to measure different characteristic (relaxation) times and correspondingly, different length scales on which dipolar motion occurs in each TPU sample under investigation, by using the molecular mobility as a probe of the structure [15]. For each sample, the shape of permittivity and dielectric loss spectra obtained from DRS are strongly related to their structure and chemistry.

Several studies on dynamics of polymer nanocomposites through dielectric spectroscopy analysis revealed that fine dispersion of the nanoparticles and their strong hydrogen bonding interaction with the polymer matrix gave rise to reduction of molecular mobility [14, 16-19]. This is in very good agreement with the results obtained from DSC and thermally stimulated depolarization currents (TSDC). The exfoliation and intercalation of organosilicate was found to influence the dielectric properties and tensile behavior of Polyetherimide (PEI) nanocomposites [20], while the works by Tsonos et al. [21] and Castagna et al. [22] emphasized the importance of DRS analysis in characterizing the microphase mixing process in the microphase separated TPU.

We have published an extensive study on a series of biomedical PDMS-based TPU nanocomposites containing organosilicates with a hydrophobic octadecyltrimethyl ammonium (bromide) (ODTMA) modification [23]. In continuation of this work, the present study aims to investigate the effect of organoclay addition on the molecular dynamics of the same E5-325 TPU system, in an attempt to develop an in-depth understanding of chemistry and molecular dynamics relationships at play in these materials. Although some research using dielectric spectroscopy on TPU has been reported, the present work is the first time that the dynamics have been investigated on the PDMS-based TPU nanocomposites, where all of the important details of mechanical properties and morphology are also known.

\section{Materials and Methods}

\section{Materials}

ElastEon E5-325 TPU consists of a $1000 \mathrm{~g} / \mathrm{mol}$ poly(dimethylsiloxane) (PDMS) and 700 $\mathrm{g} / \mathrm{mol}$ poly (hexamethylene oxide) (PHMO) mixed soft segment in a 98:2 (w/w) ratio, and a hard segment composed of alternating 4,4'-methylene diphenyl diisocyanate (MDI) and 1,4 butanediol (BDO) sequences. The hard segment concentration is $32.5 \mathrm{wt} \%$. This TPU was supplied by AorTech Biomaterials Pty Ltd. Hectorite (Lucentite SWN), which is a synthetic trioctahedral smectite was supplied by Kobo Products, Inc. It is a white powder with the chemical formula $\mathrm{Na} 0.66\left[\mathrm{Si}_{8}\left(\mathrm{Mg}_{5.34} \mathrm{Li}_{0.66}\right) \mathrm{O}_{20}(\mathrm{OH})_{4}\right]^{-0.66}$ and an average particle diameter size of approximately 30 to $50 \mathrm{~nm}$. Fluoromica (Somasif ME100), which is a synthetic mica (tetrasilicic trioctahedral fluoromica) was supplied by Kobo Products, Inc. It is a white fine powder having an average platelet size of approximately $650 \mathrm{~nm}^{23}$ with the chemical formula $\mathrm{Na}_{0.66} \mathrm{Mg}_{2.68}\left(\mathrm{Si}_{3.98} \mathrm{Al}_{0.02}\right) \mathrm{O}_{10.02} \mathrm{~F}_{1.96}$. This comparison of nanosilicate aspect ratio is an important part of this study. The surface modification 
of the nanosilicate was performed by exchanging with octadecyltrimethyl ammonium (bromide) (ODTMA) using a previously published method [23, 24].

\section{Sample preparation}

TPU nanocomposites with two different types of ODTMA modified nanosilicates; ME100 (ME) and Lucentite (Lu) were prepared in $2 \mathrm{wt} \%$ and $4 \mathrm{wt} \%$ compositions. The TPU was prepared as a $5 \mathrm{wt} \%$ solution in dimethylacetamide (DMAc), while the appropriate nanosilicate was prepared as a $5 \mathrm{wt} \%$ solution in toluene. To ensure dispersion, the nanosilicate was placed in a small glass bottle in an ultrasonic bathfor approximately 1 hour. The sample was then dispersed further by a high energy ultrasonic probe for two minutes of exposure, and then followed by another hour of dispersion in the ultrasonic bath. These steps were done to ensure a high level of swelling in the nanoclays before homogenizing. The dispersed nanosilicate was added to solution of TPU in DMAc. This was done to give a final composition of 2 and $4 \mathrm{wt} \%$ of nanofiller loading in the TPU. The combined solution was then mixed vigorously for 1 minute in a high-shear homogenizer, followed by stirring overnight at room temperature with a magnetic stirrer. The solution was then poured into a glass mould. The films were dried at $60^{\circ} \mathrm{C}$ for approximately 72 hours under a nitrogen purge. The solvent cast films were then annealed under vacuum at $85^{\circ} \mathrm{C}$ for approximately 5 hours and left to age for at least a week prior to testing. In subsequent discussion, these nanocomposites are referred to as $2 \mathrm{MEO}, 4 \mathrm{MEO}, 2 \mathrm{LuO}$ and $4 \mathrm{LuO}$. The initial number denotes the nanosilicate wt $\%$ loading $(2$ or 4$)$ in the TPU. The first two letters represent

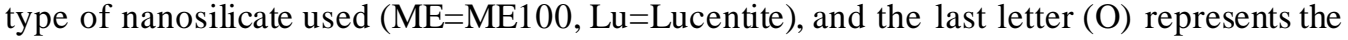
ODMTA surface modification.

\section{Broadband Dielectric Relaxation Spectroscopy (DRS)}

Dielectric constant $\left(\varepsilon^{\prime}\right)$ and loss $\left(\varepsilon^{\prime \prime}\right)$ were collected isothermally using a Novocontrol $\mathrm{GmbH}$ Concept 40 broadband dielectric spectrometer in the frequency domain from $0.01 \mathrm{~Hz}-10$ $\mathrm{MHz}$ in the range of -100 to $120^{\circ} \mathrm{C}$. Temperature stability was controlled within $\pm 0.5^{\circ} \mathrm{C}$. Sample films were sandwiched between brass electrodes of $10 \mathrm{~mm}$ diameter.

The relaxation time $\tau_{\mathrm{HN}}$ was obtained by fitting the isothermal dielectric loss $\varepsilon^{\prime \prime}(f)$ curves with the Havriliak-Negami (HN) function. The sum of multiple HN functions and a dc loss contribution were used to fit the experimental loss curves [25] :

$$
\varepsilon^{*}(\omega)=\varepsilon^{\prime}(\omega)-i \varepsilon^{\prime \prime}(\omega)=\varepsilon_{\infty}-i \frac{\sigma_{0}}{\left(\varepsilon_{0} \omega\right)^{s}}+\sum \frac{\Delta \varepsilon}{\left[1+\left(i \tau_{H N} \omega\right)^{\alpha}\right]^{\gamma}}
$$

where $\varepsilon^{*}, \varepsilon^{\prime}$, and $\varepsilon^{\prime \prime}$ are the complex, real and imaginary components of the dielectric permittivity, respectively; the dielectric relaxation strength $\Delta \varepsilon=\varepsilon_{\infty}-\varepsilon_{s}$, where $\varepsilon_{\infty}$ and $\varepsilon_{s}$ are the dielectric constants at limiting high and low frequencies, respectively. $\sigma_{0}$ is the dc conductivity $(\mathrm{S} / \mathrm{cm}) ; \omega$ is the angular frequency; $\tau_{\mathrm{HN}}$ is the characteristic relaxation time; and $\alpha$ and $\gamma$ are shape parameters, indicative of the breadth of the relaxation and peak asymmetry, respectively. The exponents characterize the conduction process. The frequency maxima $f_{\max }$ can be calculated from the following equation [26]:

$$
f_{\text {max }}=\frac{1}{2 \pi \tau_{H N}}\left[\frac{\sin \left(\frac{\alpha \pi}{2+2 \gamma}\right)}{\sin \left(\frac{\alpha \gamma \pi}{2+2 \gamma}\right)}\right]^{1 / \alpha}
$$

To remove the impurity ion motion above $\mathrm{T}_{\mathrm{g}}$, the dielectric loss was calculated from the first derivative of $\varepsilon^{\prime}$ which has been shown for relatively broad relaxations to provide a good estimate of the conduction-free dielectric loss [27]: 


$$
\varepsilon_{d e r}^{\prime \prime}=-\frac{\pi}{2} \frac{\partial \varepsilon^{\prime}(f)}{\partial \ln f}
$$

\section{Results and Discussion}

Dielectric Relaxation Spectroscopy (DRS) of E5-325 TPU matrix and nanocomposites

Generally, the dielectric spectrum of the TPU over broad frequency range shows multiple relaxations, depending on the structure and temperature, where each process is represented by a peak in dielectric loss $\left(\varepsilon^{\prime \prime}\right)$ in the temperature and frequency domains and associated step change in dielectric constant $\left(\varepsilon^{\prime}\right)$ as a function of frequency (f) at a fixed temperature [28-30]. Changes in the strength, position and shape of the spectra can be interpreted in terms of varying degree of interaction between the TPU soft phase and the organosilicate nanofillers. Therefore, the effect of organosilicate aspect ratio and loading on the molecular dynamics of the complex PDMS based TPU system was investigated. In all samples, the dielectric relaxation spectrum was determined to study the molecular dynamics of the neat host TPU and nanocomposites. The morphology of these E5-325 TPU and nanocomposites has been extensively characterized in previous DMTA, DSC, XRD, SAXS and TEM analysis [23] and the results were utilized to aid in interpretation of the molecular dynamics obtained from the DRS studies.

Over the measured temperature and frequency range, three relaxations were observed for all samples referred as $\alpha_{1}, \alpha_{2}$, and $\alpha_{3}$ as shown in Figure 1. The relaxation frequency of all processes is fit well by the Vogel-Fulcher-Tammann(VFT) equation (eq. 4) [31] as displayed in Figure 2. The fitting parameters are provided in Table 1.

$$
f 0 \exp \left(-\frac{D T_{0}}{T-T_{0}}\right)_{\text {max }}
$$

where $f_{0}$ is the exponential pre-factor, $\mathrm{T}_{0}$ is the Vogel temperature below which segments become immobile. $D$ is the strength parameter, quantifying the divergence from Arrhenius temperature dependence [31]. Lower $D$ corresponds to a higher fragile system or less Arrhenius-like behavior.

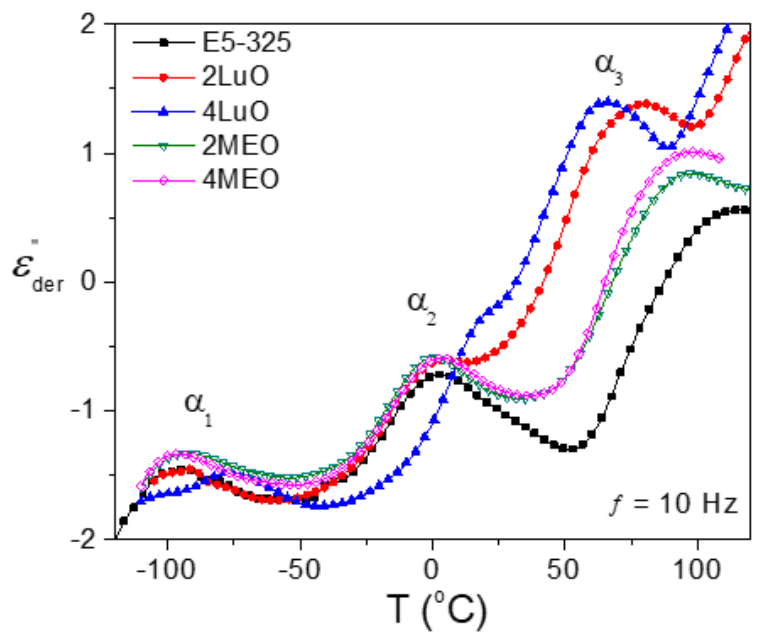

Fig. 1. Derivative loss spectra of E5-325, 2LuO, 4LuO, $2 \mathrm{MEO}$ and $4 \mathrm{MEO}$ vs temperature at $10 \mathrm{~Hz}$ 
According to the VFT behavior, the $\alpha_{1}$ relaxation displayed by the E5-325 TPU at low temperature is in a similar location observed using DMTA at $-85^{\circ} \mathrm{C}$, is assigned to the segmental relaxation of the PDMS phase [23]. The $\alpha_{2}$ relaxation occurred above the soft microphase transition temperature as determined from DMTA. Therefore, this relaxation was attributed to the segmental relaxation of the mixed soft segments. Based on the segmental microphase separation in E5-325 and its nanocomposites observed from SAXS, the $\alpha_{3}$ process is proposed to the process associated with Maxwell-Wagner-Sillars (MWS) interfacial polarization [21,22,33] arising from the accumulation of ions at the interface of soft and hard segments, which are having different dielectric constant and conductivity.

(a)

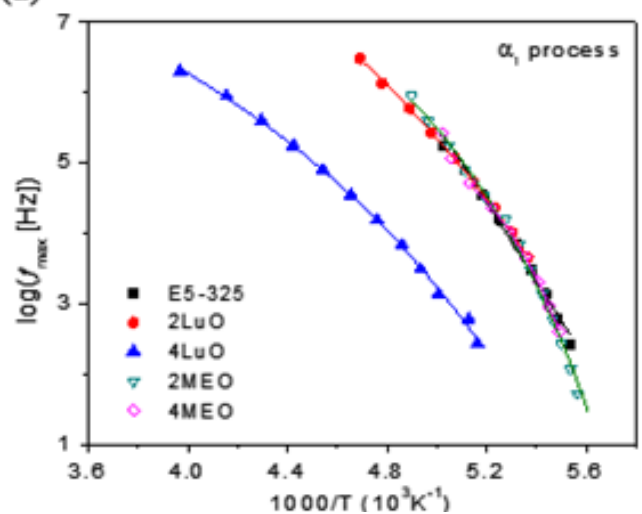

(b)

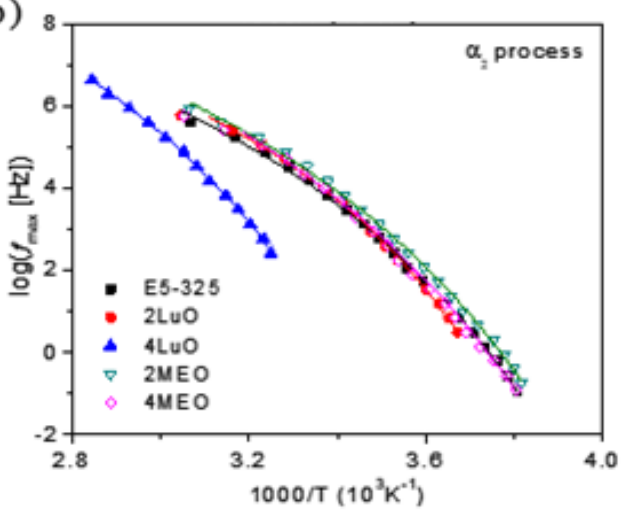

(c)

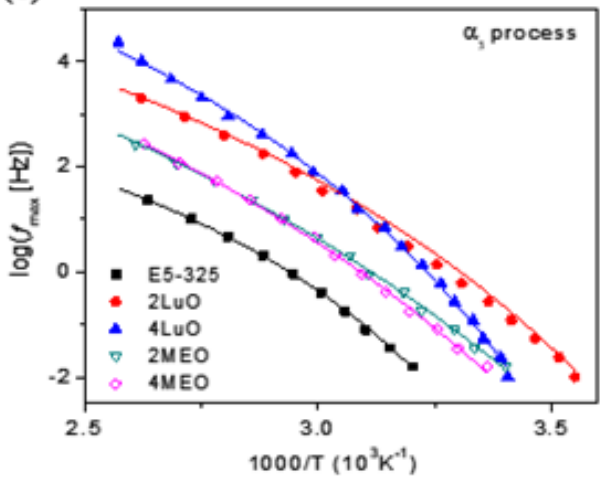

Fig. 2. Relaxation frequencies of the (a) $\alpha_{1}$, (b) $\alpha_{2}$ and (c) $\alpha_{3}$ process for E5-325, 2LuO, 4LuO, 2MEO and 4MEO. Lines indicate the VFT fit

Table 1. VFT fit parameters for the $\alpha_{1}$, and $\alpha_{2}$ processes for E5-325, 2LuO, 4LuO, 2MEO and 4MEO

\begin{tabular}{lcccccccc}
\hline & \multicolumn{3}{c}{$\alpha_{1}$ process } & & \multicolumn{3}{c}{$\alpha_{2}$ process } \\
\cline { 2 - 4 } \cline { 7 - 8 } Sample & $\log \mathrm{f}_{0}(\mathrm{~Hz})$ & $D$ & $\mathrm{~T}_{0}(\mathrm{~K})$ & & $\log \mathrm{f}_{0}(\mathrm{~Hz})$ & $D$ & $\mathrm{~T}_{0}(\mathrm{~K})$ \\
& & & & & & & & \\
\hline E5-325 & 10.9 & 4.9 & 144 & & 11.7 & 7.8 & 207 \\
2LuO & 10.9 & 5.0 & 143 & & 11.0 & 5.4 & 226 \\
4LuO & 10.2 & 7.6 & 136 & & 13.3 & 7.5 & 236 \\
2MEO & 10.9 & 4.2 & 149 & & 12.1 & 8.2 & 205 \\
4MEO & 10.2 & 3.9 & 149 & & 12.4 & 8.5 & 207 \\
\hline
\end{tabular}


Also, worth noting from Figure 1 is the difference in curve shapes between different polymer nanocomposite samples. TPU nanocomposites incorporating low aspect ratio LuO exhibitdifferent dielectric spectra as compared to those with high aspect ratio MEO. Furthermore, the $\mathrm{LuO}$ nanocomposite system demonstrates a large change in the relaxation behavior when the filler content is increased from 2 to $4 \mathrm{wt} \%$. In the $4 \mathrm{wt} \%$ sample, the $\alpha_{1}$ and $\alpha_{2}$ relaxations are shifted to higher temperature, whereas the $\varepsilon$ " value was found to be lower in the glassy region and much higher in the rubbery region. A higher value of derivative ( $\varepsilon$ "der) for $\alpha_{2}$ means a higher polarity of the relaxing units contributing to the mixed-phase $\left(\alpha_{2}\right)$ relaxation. Based on previous DMTA and DSC data, we have postulated that the LuO nanofiller is the most effective at compatibilising the hard and soft TPU segments, resulting in phase mixing [5, 23]. As the hard segments are strong dipolar units, a contribution of the dissolved hard segments in the continuous soft phase and in the interphases to the dielectric losses should be expected. The increased addition of $\mathrm{LuO}$ from $2 \mathrm{wt} \%$ to $4 \mathrm{wt} \%$ also causes a shift of $\mathrm{T} \alpha_{2}$ to higher values, with a broader and weaker peak. This suggests that the dissolution of the hard segments into the soft segments impedes the molecular mobility of the soft phase. After reaching $\alpha_{2}$, a larger upturn in $\varepsilon$ "der approaching $\alpha_{3}$ process was observed in $2 \mathrm{LuO}$ and $4 \mathrm{LuO}$ with respect to other materials. This transition occurs in the region previously identified and associated with the hard domain $\mathrm{T}_{\mathrm{g}}$ by DSC [5]. Thus, the larger increase in $\varepsilon$ "der should be related to the presence of greater hydrogen bonding between the hard segments, forming a more well-ordered and interconnected hard domain population $[5,23]$.

While the nanocomposites incorporating $\mathrm{LuO}$ show a large change in relaxation behaviour with increasing filler loading, nanocomposites incorporating MEO on the other hand do not. The dielectric spectra of $2 \mathrm{MEO}$ and $4 \mathrm{MEO}$ are very similar indicating similar segmental processes, but with a slight increase in dielectric loss accompanying an increase in organosilicate content from $2 \mathrm{wt} \%$ to $4 \mathrm{wt} \%$.

Figure $2 \mathrm{a}$ and $2 \mathrm{~b}$ revealed that the segmental relaxation times of the $\alpha_{1}$ and $\alpha_{2}$ processes of all samples except for $4 \mathrm{LuO}$ are almost the same. The $\alpha_{1}$ and $\alpha_{2}$ processes of $4 \mathrm{LuO}$ are slower than others, attributed to greater dissolution of hard segments as well as the better dis persion of small size hard microdomains in the continuous soft phase which hinders the mobility the soft phase. This is in agreement with highest storage modulus and soft microphase $T_{\mathrm{g}}$ observed from DMTA [5, 23].

The migration of ions, accumulated at the interface of soft and hard segments for all nanocomposites are faster than that of neat host TPU as seen in Figure 2c. The rate of ion accumulation is in the order of $4 \mathrm{LuO}>2 \mathrm{LuO}>4 \mathrm{MEO} \approx 2 \mathrm{MEO}>\mathrm{E} 5-325$, indicating faster ion motions in the E5-325 containing low aspect ratio hectorite than those containing high aspect ratio fluoromica. LuO has previously proved to act as interfacial compatiblizer between TPU soft and hard segments by promoting phase mixing [5]. The faster ion motions in the MWS region of the E5-325 containing LuO organosilicates originate from the motion of the soft segments containing polar hard segments, which were dissolved in the continuous soft segments. This phenomenon can also be observed from a substantial increase in number of dipoles in both $2 \mathrm{LuO}$ and $4 \mathrm{LuO}$ samples when the temperature approaches the $\alpha_{3}$ (MWS) process (Figure 4).

Fragility reflects the degree of intermolecular coupling. It is believed that as intermolecular coupling becomes stronger, the material becomes more fragile [34-36]. Zhang et al. [34] reported the increase of fragility with increasing intermolecular hydrogen bonding between poly(4-vinylphenol)-poly(vinyl ethyl ether)[PVPh/PVEE] blends. Based on Table 1, the fragility of LuO nanocomposites in the phase-mixed ( $\alpha_{2}$ region) is greater than the neat host TPU and other materials. It is reasonable to assume that this is a consequence of stronger intermolecular bonding, which couples the relaxation of the hard and soft segments.

The breadths of the $\alpha_{1}$ relaxations of $2 \mathrm{LuO}, 4 \mathrm{LuO}, 2 \mathrm{MEO}$ and $4 \mathrm{MEO}$ are slightly broader than that of E5-325, suggesting that the fillers all induce a small degree of dynamic heterogeneity on PDMS. Interestingly, the breadths of the $\alpha_{2}$ relaxations of $2 \mathrm{LuO}$ and $4 \mathrm{LuO}$ are 
slightly broader than other materials, indicating a higher distribution of the $\alpha_{2}$ relaxation time, due to higher heterogeneity of the soft microphase as a result of phase mixing, as suggested by Tsonos and co-workers [21].
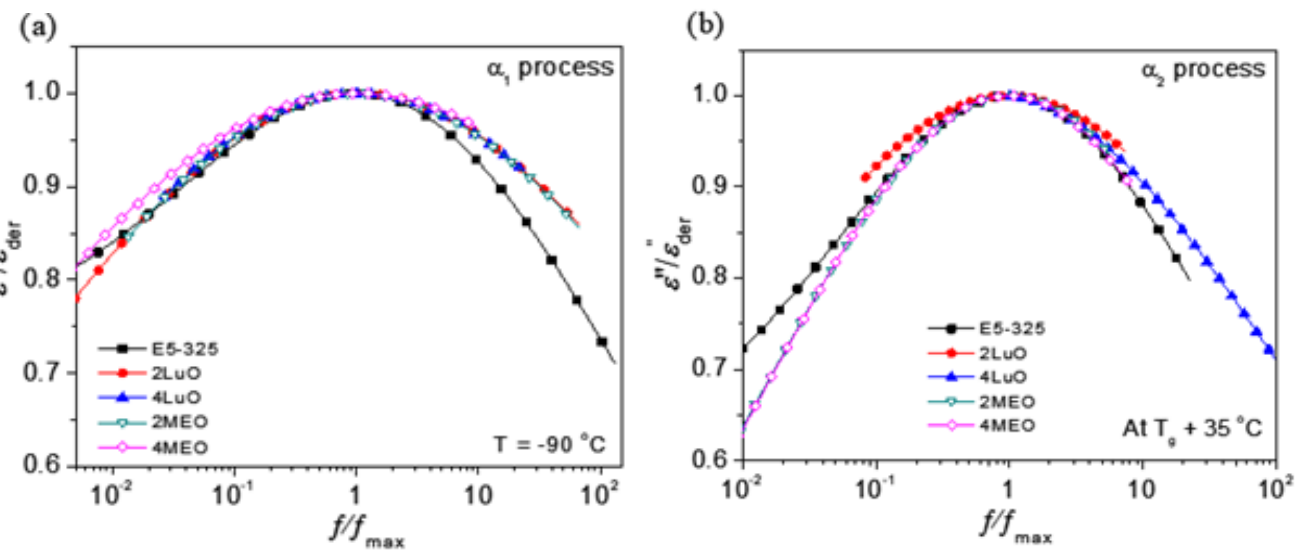

Fig. 3. Norma lized segmental loss of the (a) $\alpha_{1}$ and (b) $\alpha_{2}$ process forE5-325, 2LuO, $4 \mathrm{LuO}, 2 \mathrm{MEO}$ and $4 \mathrm{MEO}$ at $\mathrm{T}=-80^{\circ} \mathrm{C}$

Table 2. Dielectric Relaxation Strength of the $\alpha_{1}, \alpha_{2}$ and $\alpha_{3}$ process

\begin{tabular}{lccc}
\hline Sample & $\begin{array}{r}\Delta \varepsilon\left(\alpha_{1}\right) \\
\text { at }-80{ }^{\circ} \mathrm{C}\end{array}$ & $\begin{array}{c}\Delta \varepsilon\left(\alpha_{2}\right) \\
\text { at } \mathrm{T}_{\mathrm{g}}+35{ }^{\circ} \mathrm{C}\end{array}$ & $\begin{array}{c}\Delta \varepsilon\left(\alpha_{3}\right) \\
\text { at } 90{ }^{\circ} \mathrm{C}\end{array}$ \\
\hline E5-325 & 0.4 & 1.7 & 19 \\
$2 \mathrm{LuO}$ & 0.6 & 2.4 & 57 \\
$4 \mathrm{LuO}$ & 0.5 & 5.5 & 43 \\
2MEO & 0.7 & 2.1 & 33 \\
4MEO & 0.9 & 2.2 & 36 \\
\hline
\end{tabular}

The dielectric relaxation strengths $\Delta \varepsilon$ of $\alpha_{1}, \alpha_{2}$ and $\alpha_{3}$ processes at $-80, \mathrm{~T}_{\mathrm{g}}+35$ and 90 ${ }^{\circ} \mathrm{C}$, respectively are given in Table 2 . For all TPU nanocomposites, $\Delta \varepsilon\left(\alpha_{1}\right), \Delta \varepsilon\left(\alpha_{2}\right)$ and $\Delta \varepsilon\left(\alpha_{3}\right)$ values are higher than that of the neat host TPU. The increase of $\Delta \varepsilon\left(\alpha_{1}\right)$ and $\Delta \varepsilon\left(\alpha_{2}\right)$ with the organosilicate addition indicates that there are some dielectrically active mobile molecules, present in the TPU nanocomposites, possibly attached to the TPU soft segments. It is reasonable to assume that these dielectrically active molecules may originate from the alkylammonium ions on the nanofiller surface.

A dramatically increases of $\Delta \varepsilon\left(\alpha_{3}\right)$ (MWS process) is caused by the higher number of mobile ions in TPU nanocomposites respective to neat host TPU, due to the entrapment of more charges in the TPU matrix, especially between clay platelets [37].

It is also worth noting that the dielectric strength of MEO nanocomposites in $\alpha_{2}$ and $\alpha_{3}$ process is lower than that of $\mathrm{LuO}$ nanocomposites. The result could be attributed to the aggregation of MEO, since aggregation would greatly reduce the dielectric strength of the materials [38]. The aggregation formation can be observed from a large MEO tactoids present in the TPU matrix as obtained from TEM analysis [5]. Highest $\Delta \varepsilon\left(\alpha_{3}\right)$ was achieved by $2 \mathrm{LuO}$, as a result of better polarity contrast between the soft and hard domains, due to the existence of a more interconnected hard domain population in this nanocomposite system, as previously characterized by in-situ strained synchrotron SAXS and DSC [5]. 


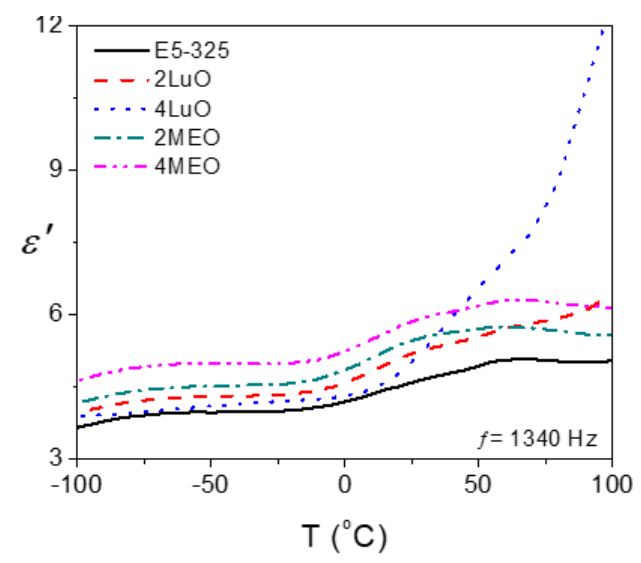

Fig. 4. Dielectric constant of E5-325,2LuO, 4LuO, $2 \mathrm{MEO}$ and $4 \mathrm{MEO}$ vs temperature at $1340 \mathrm{~Hz}$

Figure 4 presents the dielectric constant $\varepsilon^{\prime}$ of neat host TPU and nanocomposites as a function of temperature at $1340 \mathrm{~Hz}$. It clearly shows that the $\varepsilon^{\prime}$ of all nanocomposites is higher that that of the neat host TPU and that this disparity increases with temperature. The $\varepsilon^{\prime}$ increases in the order of $4 \mathrm{MEO}>2 \mathrm{MEO}>2 \mathrm{LuO}>4 \mathrm{LuO}>\mathrm{E} 5-325$, indicating an increase in number of dipoles in TPU samples incorporating organosilicates. This is due to the higher dielectric constant of the organosilicate with respect to the polymer [37,39]. This trend was also observed by Pissiset al. [14, 18] in SBR/silica nanocomposite and Dwivedi et al. [20] in polyetherimide/organosilicate nanocomposites. The dielectric constant of a material is related to the polarization mechanism. The morphology of the TPU [37,40] and organosilicates dispersion [37] would have an influence on the polarization behaviour. For the neat host TPU and nanocomposites, poorer polarization occurred at cryogenic temperature, resulting in a lower dielectric constant. However, rapid increase of the dielectric constant began near their mixed soft phase transition temperature, where additional polarization occurs with an increase of soft segment mobility. After reaching $\sim 25^{\circ} \mathrm{C}$, the dielectric constant of both $4 \mathrm{LuO}$ and $2 \mathrm{LuO}$ was seen to increase further, while th at of $2 \mathrm{MEO}$ and 4MEO started to level off. A sharp increase of $\varepsilon^{\prime}$ in the $4 \mathrm{LuO}$ sample might be due to interfacial polarization or the accumulation of charges at the interphase between the mixed phase matrix and the organosilicates. Moreover, TEM images of LuO nanocomposites demonstrate greater organosilicate dispersion and exfoliation in the TPU matrix compared to MEO nanocomposites, showing more individual nanosilicate layers [5]. These factors should result in higher dielectric constant, as polarization would increase due to the effect of multi-interface polarization $[39,40]$.

\section{Conclusions}

In summary, we have successfully investigated molecular dynamics of the E5-325 TPU and nanocomposites in relation to their structure and morphology by using dielectric spectroscopy. Several interesting results were obtained with each of the systems studied and discussed in relation to results obtained previously by using other techniques such as DMTA, DSC, TEM and SAXS. There are several findings that can be emphasized here. These are; (i) Dielectric spectra of well-characterized E5-325 TPU and nanocomposites exhibit three dipolar relaxation processes, which are referred as $\alpha_{1}, \alpha_{2}$, and $\alpha_{3}$. (ii) Aspect ratio and loading of the organosilicates, and TPU-nanofiller interactions are reflected in the characteristics of the cooperative segmental relaxation. (iii) The segmental relaxation time and temperature of $\alpha_{1}, \alpha_{2}$, and $\alpha_{3}$ process of the TPU matrix are significantly altered by the presence of the hydrophobic low aspect ratio organo-hectorite $(\mathrm{LuO})$, where significant changes in the dynamics were 
observed. (iv) Dielectric data confirm the existence of phase mixing in the E5-325 TPU containing 4wt\% LuO, which is in good agreement with the DMTA, DSC and SAXS results presented in our previous paper.

\section{Acknowledgements}

The authors thank Aortech Biomaterials Pty Ltd and Cochlear Ltd for research materials and financial support. We also appreciate Mr Hyeok Choi from Pennsylvania State University for the expert DRS training he provided. Azlin F. Osman expresses her appreciation to the Ministry of Higher Education Malaysia and UQ Graduate School for financial support.

\section{References}

[1] M. Szycher, Szycher's handbook of polyurethanes, 2nd Edition. CRC Press, Boca Raton, Fluorida, 2012,pp. 354.

[2] M. V. Pergal, M. Balaban, B. Dojčinović, D. Manojlović., Thermoplastic polyurethane nanocomposites in Thermoplastic Composites: Emerging Technology, Uses and Prospects, Edited by Ritter, E., Nova Science Publishers, Inc., 2017, pp. 1-60

[3] J. T. Haponiuk, K. Formela, K. "PU Polymers, Their Composites, and Nanocomposites: State of the Art and New Challenges in Polyurethane Polymers: Composites and Nanocomposites", Elsevier Inc., 2017, pp. 1-20.

[4] P. Gunatillake, G. Meijs, S. Mccarthy, R. Adhikari, Poly(dimethylsiloxane)/poly(hexamethylene oxide) mixed macrodiol based polyurethane elastomers. I. Synthesis and properties, J. Appl. Polym. Sci. 2000, 76(14), 2026-2040.

[5] A. F. Osman, Y. Andriani, G.A.Edwards, T.L. Schiller, K.S. Jack, I.C. Morrow, P.J. Halley, D.J. Martin. Structure-Property Relationships in Biomedical Thermoplastic Polyurethane Nanocomposite, Macromolecules, 45 (1), 2011, 198-210.

[6] A. F. Osman, D.J. Martin, Thermoplastic polyurethane (TPU)/organo-fluoromica nanocomposites for biomedical applications: in vitrofatigue properties, IOP Conf. Ser.: Mater. Sci. Eng. 2019, 701, 012056.

[7] A. F. Osman, K. Jack, G. Edwards, D. Martin, Effect of Processing Route on the Morphology of Thermoplastic Polyurethane (TPU) Nanocomposites Incorporating Organofluoromica, Advanced Materials Research, 2014, 832, 27-32.

[8] A. R. Abdul Hamid, A. F. Osman, Z. Mustafa, S. Mandal, R. Ananthakrishnan, Tensile, fatigue and thermomechanical properties of poly(ethylene-co-vinyl acetate) nanocomposites incorporating low and high loadings of pre-swelled organically modified montmorillonite, Polymer Testing, 2020, 106426.

[9] D. K. Patel, A. K. Mahanta \& P. Maiti, Polyurethane Nanocomposites of Layered Silicates in Polyurethane Polymers: Composites and Nanocomposites, Elsevier Inc., 2017, pp. 173-189.

[10] Y. Rao, Y, Engineering of Interface in Nanocomposites Based on PU Polymers in Polyurethane Polymers: Composites and Nanocomposites, Elsevier Inc., pp. 73-133, 2017.

[11] A. A Ahmad Fauzi, A. F. Osman, M. A. A. Abdullah, S. Mandal and R. Ananthakrishnan, Ethylene vinyl acetate nanocomposites with hybrid silicate nanofillers of destabilized natural and commercial bentonites and organomontmorillonites. Journal of Vinyl \& Additive Technology, 2019, 25 (4), 396-411.

[12] A. K. Barick, D.K. Tripathy, Effect of Organoclay on the Morphology, Mechanical, Thermal, and Rheological Properties of Organophilic Montmorillonite Nanoclay Ba sed 
Thermoplastic Polyurethane Nanocomposites Prepared by Melt Blending, Polym.Eng.Sci. 2010, 50 (3), 484-498.

[13] S. Smart, D. Fania, A. Milev, G. Kannangara, M. Lu, D. Martin, The effect of carbon nanotube hydrophobicity on the mechanical properties of carbon nanotube reinforced thermoplastic polyurethane nanocomposites, Journal of Applied Polymer Science, 117(1), 2010, 24-32.

[14] P. Pissis, D. Fragiadakis, A. Kanapitsas, K. Delides, In Broadband Dielectric Relaxation Spectroscopy in Polymer Nanocomposites, Wiley Online Library: 2008; pp 12-20.

[15] F. Kremer, F. A. Schönhals, Broadband dielectric spectroscopy. Springer Verlag: 2003. Springer, Berlin, Heidelberg.

[16] D. Fragiadakis, P. Pissis, L. Bokobza, Glass transition and molecular dynamics in poly(dimethylsiloxane)/silica nanocomposites, Polymer, 2005, 46 (16), 6001-6008.

[17] D. Fragiadakis, P. Pissis, Glass transition and segmental dynamics in poly (dimethylsiloxane)/silica nanocomposites studied by various techniques, Journal of NonCrystalline Solids, 2007, 353 (47-51), 4344-4352.

[18] D. Fragiadakis, E.Logakis, P. Pissis, V.Y. Kramarenko, T. Shantalii, I. Karpova, K. Dragan, E. Privalko, A. Usenko, V. Privalko, In Polyimide/silica nanocomposites with low values of dielectric permittivity, IOP Publishing: 2005; p 139.

[19] P. F. Green, H. Oh, P. Akcora, S.K. Kumar, Structure and Dynamics of Polymer Nanocomposites Involving Chain-Grafted Spherical Nanoparticles Dynamics of Soft Matter. In García Sakai, V.; Alba-Simionesco, C.; Chen, S.-H., Eds. Springer US: 2012; pp 349-366.

[20] M. Dwivedi, A. Dixit, S. Alam, A.K. Ghosh, Dielectric and tensile behavior of nanoclay reinforced polyetherimide nanocomposites, Journal of Applied Polymer Science, 2011, 122 (2), 1040-1046.

[21] Tsonos, C.; Apekis, L.; Zois, C.; Tsonos, G. Acta materialia 2004, 52 (5), 1319-1326.

[22] A. M. Castagna, D. Fragiadakis, H. Lee, T. Choi, J. Runt, The role of hard segment content on the molecular dynamics of poly (tetramethylene oxide)-based polyurethane copolymers, Macromolecules, 44(19), 2011, 7831-7836.

[23] A. F. Osman, Y. Andriani, G. A. Edwards, T.L. Schiller, K.S. Jack, I.C. Morrow, P.J. Halley, D.J. Martin, Engineered nanofiller: Impact on the Morphology and Properties of Biomedical Thermoplastic Polyurethane Nanocomposites. RSC Advances, 2012, 2(24), 9151-9164.

[24] Y. Andriani, K.S Jack, E.P. Gilbert, G.A.Edwards, T.L. Schiller, E. Strounina, A.F. Osman, D.J. Martin, Organization of mixed dimethyldioctadecylammonium and choline modifiers on the surface of synthetic hectorite, Journal of Colloid and Interface Science, 2013, 409, 72-79.

[25] S. Havriliak, S. Negami, A complex plane analysis of $\alpha$-dispersions in some polymer systems, Journal of Polymer Science Part C: Polymer Symposia 1966, 14 (1), 99-117.

[26] K. A. Masser, J. Runt, Dynamics of polymers blends of a strongly interassociationg homopolymer with Poly(Vinul methyl ether) and Poly(2-vinylpyrifine), Macromolecules 2010, 43(15), 6414-6421.

[27] P. Steeman, J. Van Turnhout, Fine structure in the parameters of dielectric and viscoelastic relaxations, Macromolecules, 1994, 27 (19), 5421-5427.

[28] Z. S. Petrović, I. Javni, A. Waddon, G. Bánhegyi, Structure and properties of polyurethanesilica nanocomposites, Journal of Applied Polymer Science, 76 (2), 2000, 133-151.

[29] S. Oprea, Degradation of crosslinked poly(ester-urethanes) elastomers in distilled water: Influence of hard segment, Journal of Applied Polymer Science, 2011, 124 (2), 1059-1066. 
[30] C. Tsonos, L. Apekis, K. Viras, L. Stepanenko, L. Karabanova, L. Sergeeva, Electrical and dielectric behavior in blends of polyurethane-based ionomers, Solid state ionics, 2001, 143 (2), 229-249.

[31] D. Fragiadakis, S. Dou, R. H. Colby, J. Runt, Molecular mobility, ion mobility, and mobile ion concentration in poly( ethylene oxide)-based polyurethane ionomers, Macromolecules, 2008, 41 (15), 5723-5728.

[32] A. Saiter, J. Saiter, J. Grenet, Cooperative rearranging regions in polymeric materials: Relationship with the fragility of glass-forming liquids, European polymer journal, 2006, 42 (1), 213-219.

[33] P. Atorngitjawat, R.J. Klein, J, Runt, Dynamics of sulfonated polystyrene copolymers using broadband dielectric spectroscopy, Macromolecules, 2006, 39 (5), 1815-1820.

[34] S. Zhang, P. C. Painter, J. Runt, Dynamics of Polymer Blends with Intermolecular Hydrogen Bonding: Broad-Band Dielectric Study of Blends of Poly(4-vinyl phenol) with Poly(vinyl acetate) and EVA70, Macromolecules, 2002, 35 (25), 9403-9413.

[35] H. Lee, D. Fragiadakis, D. Martin, A. Milne, J. Milne, J. Runt, Dynamics of uniaxially oriented elastomers using broadband dielectric spectroscopy, Macromolecules, 2010, 43(7), 3125-3127.

[36] C. A. Angell, Formation of glasses from liquids and biopolymers, Science, 1995, 267, (5206), 1924.

[37] M. Razzaghi-Kashani, N. Gharavi, S. Javadi, The effect of organo-clay on the dielectric properties of silicone rubber, Smart Materials and Structures, 17, 2008, 065035.

[38] Y. H. Zhang, Z. M. Dang, S. Y. Fu, J. H. Xin; J. G. Deng, J. Wu, S. Yang, L. F. Li, Q.Yan, Dielectric and dynamic mechanical properties of polyimide-clay nanocomposite films, Chemical physics letters, 2005, 401 (4-6), 553-557.

[39] L. Y. Jiang, C. M. Leu, K. H. Wei, Layered silicates/fluorinated polyimide nanocomposites for advanced dielectric materials applications, Advanced Materials, 2002, 14 (6), 426-429.

[40] Y. H. Zhang, Z. M. Dang, J. H. Xin, W.A. Daoud, J. H. Ji, Y. Liu, B. Fei, Y. Li, J. Wu, S. Yang, Dielectric properties of polyimide-mica hybrid films, Macromolecular rapid communications, 26 (18), 2005, 1473-1477. 